\title{
Korovkin Approximation Theorem with $\Omega$ Striped
}

\author{
Malik Saad Al-Muhja ${ }^{1,2}$, Mohammad Mursaleen ${ }^{3}$, Masnita Misiran ${ }^{1}$, Zurni B. Omar ${ }^{1} \&$ Sui Yang Khoo ${ }^{4}$ \\ ${ }^{1}$ Department of Mathematics and statistics, School of Quantitative Sciences, College of Arts and Sciences, Universiti \\ Utara Malaysia, 06010 Sintok, Kedah, Malaysia \\ ${ }^{2}$ Department of Mathematics and Computer Application, College of Sciences, University of Al-Muthanna, Samawa \\ 66001, Iraq \\ ${ }^{3}$ Department of Mathematics, Aligarh Muslim University, Aligarh 202002, India \\ ${ }^{4}$ Faculty of Science Engineering and Built Environment, Deakin University, Geelong VIC 3220, Australia \\ Correspondence: Malik Saad Al-Muhja, Department of Mathematics and statistics, School of Quantitative Sciences, \\ College of Arts and Sciences, Universiti Utara Malaysia, 06010 Sintok, Kedah, Malaysia. \\ Malik Saad Al-Muhja (malik@mu.edu.iq), Mohammad Mursaleem (mursaleen.mm@amu.ac.in) \\ Masnita Misiran (masnita@uum.edu.my),Zurni Omar (zurni@uum.edu.my), \\ Sui Yang Khoo (sui.khoo@deakin.edu.au)
}

Received: September 29, 2017 Accepted: October 17, $2017 \quad$ Online Published: November 18, 2017

doi:10.5539/jmr.v10n1p20

URL: https://doi.org/10.5539/jmr.v10n1p20

\begin{abstract}
In this paper, we discuss some theorem reached M. Mursaleen, there are several properties of statistical lacunary summability presented (Mursaleen, M. \& Alotaibi, A., 2011; Mursaleen, M. \& Alotaibi, A., 2011; Edely, O. H. \& Mursaleen, M., 2009). This is concerned the motivate to narrowly delineated context denoted by $\Omega$ striped usage in prove our theorem (theorem A). We introduce some piecewise polynomial functions (Kopotun, K. A., 2006) and some results Korovkin theorem.
\end{abstract}

Keywords: Piecewise polynomial functions, Statistical lacunary summability, Strongly $\theta_{\mathrm{q}}$-convergent, Korovkin type theorem

2010 Math. Sub. Classification: 41A10, 41A25, 41A36, 40A30

\section{Introduction and Main Results}

The aim of this paper a completed the striped used in many area of Korovkin theorem (Mursaleen, M. \& Alotaibi, A., 2011; Al-Muhja, M., 2015).

We will need accept the following:

Let $K \subseteq \mathbb{N}$. Then $\delta_{\theta}(K)=\lim _{r} \frac{1}{h_{r}}\left|\left\{k_{r-1}<i<k_{r}: i \in K\right\}\right|$ is said to be $\theta$-density of $K$.

Definition 1.1 (Fridy, J. A. \& Orhan, C., 1993) A sequence $x=\left(x_{k}\right)$ is said to be lacunary statistically convergent to $L$, if for every $\epsilon>0$, the set $K_{\epsilon}:=\left\{k \in \mathbb{N}:\left|x_{k}-L\right| \geq \epsilon\right\}$ has $\theta$-density zero, i.e. $\delta_{\theta}\left(K_{\epsilon}\right)=0$. In this case we write $S_{\theta}-\lim x=L$. That is, $\lim _{r}\left|\left\{k_{r-1}<i<k_{r}:\left|x_{k}-L\right| \geq \epsilon\right\}\right|=0$. In this case we write $S_{\theta}-\lim _{i} x_{i}=L$, and we denote the set of all lacunary statistically convergent sequence by $S_{\theta}$.

Definition 1.2 (Mursaleen, M. \& Alotaibi, A., 2011) Let $h=\left(h_{r}\right)$ be a non-decreasing sequence of positive numbers tending to $\infty$ such that $h_{r+1} \leq h_{r}+1, x_{1}=0$. The generalized de la Vallee-Poussin mean is defined by $t_{r}(x)=$ $\frac{1}{h_{r}} \sum_{j \in I_{r}} x_{j}$ where $I_{r}=\left[r-h_{r}+1, r\right]$.

Definition 1.3 (Mursaleen, M. \& Alotaibi, A., 2011) A sequence $x=\left(x_{k}\right)$ is said to be $\theta$-summable to $L$, if $\lim _{r} t_{r}(x)=L$.

Definition 1.4 (Mursaleen, M. \& Alotaibi, A., 2011) A sequence $x=\left(x_{k}\right)$ is said to be statistically lacunary summable (or statistically $\theta$-summable) to $L$, if for every $\epsilon>0$, the set $K_{\epsilon}:=\left\{r \in \mathbb{N}:\left|t_{r}(x)-L\right| \geq \epsilon\right\}$ has natural density zero, 
i.e., $\delta\left(K_{\epsilon}(\theta)\right)=0$. That is, $\lim _{n} \frac{1}{n}\left|\left\{r \leq n:\left|t_{r}(x)-L\right| \geq \epsilon\right\}\right|=0$. In this case we write $S_{\theta}-\lim x=L$. We denote the set of all statistically lacunary summable sequences by $\theta_{S}$.

Definition 1.5 (Mursaleen, M. \& Alotaibi, A., 2011) A sequence $x=\left(x_{k}\right)$ is said to be strongly $\theta_{q}$-convergent $(0<q<\infty)$ to the limit $L$, if $\lim _{r} \frac{1}{h_{r}} \sum_{j \in I_{r}}\left|x_{j}-L\right|^{q}=0$, and we write it as $x_{k} \rightarrow L\left[C_{\theta}\right]_{q}$. In this case $L$ is called the $\left[C_{\theta}\right]_{q}$-limit of $x$. We denote the set of all strongly $\theta_{q}$-convergent sequences by $L\left[C_{\theta}\right]_{q}$.

Let $\mathcal{S}_{r}\left(\mathrm{z}_{n}\right)$ be the space of all piecewise polynomial functions of degree $r$ ( order $r+1$ ), with the knots

$$
\mathrm{z}_{n}=\left(\mathrm{z}_{i}\right)_{i=0}^{n},-1=\mathrm{z}_{0}<\mathrm{z}_{1}<\cdots<\mathrm{z}_{n-1}<\mathrm{z}_{n}=1 .
$$

Definition 1.6 (Al-Muhja, M., 2015) A spline $s$ in $G_{s} /\left\{I_{e}\right\}$ is said to be homogeneous of degree $\lambda \in \mathcal{R}$ if $s \circ \gamma_{\tau}=\tau^{\lambda} s$ for $\tau>0$.

Definition 1.7 (Al-Muhja, M., 2015) A distribution $\sigma$ on $G_{s}$ is said to be homogeneous of degree $\lambda$ if $\left\langle\sigma,\left(\tau^{-\sum_{i=1}^{\eta} d_{i}}\right) s \circ \gamma_{\tau-1}\right\rangle=\tau^{\lambda}\langle\sigma, s\rangle$, for $s \in G_{s}, d_{i} \in \mathcal{R}, \eta \in \mathbb{N}$ and $\tau>0$.

Definition 1.8 (Al-Muhja, M., 2015) A linear differential operator $\Upsilon$ on $G_{s}$ is said to be homogeneous of degree $\lambda$ if $\Upsilon\left(s \circ \gamma_{\tau}\right)=\tau^{\lambda}(\Upsilon s) \circ \gamma_{\tau}$, for any $s \in G_{s}$ and $\tau>0$.

Lemma 1.9 (Al-Muhja, M., 2015) Let $A=\left(a_{j n}\right)$ be nonnegative regular summability matrix. For all $s \in G_{s} /\left\{I_{e}\right\}$, satisfied ((Al-Muhja, M., 2015) equation (3)), and $Y_{j}$ a sequence of positive linear operators, we have $Y_{j}: G_{s} /\left\{I_{e}\right\} \rightarrow$ $G_{s} /\left\{I_{e}\right\}$, homogeneous group.

Theorem 1.10 (Al-Muhja, M., 2015) Let $A=\left(a_{j n}\right)$ be nonnegative regular summability matrix, and let $Y_{j}$ be a sequence of positive linear operators from $\mathcal{S}_{r}\left(z_{n}\right)$ into $\mathcal{S}_{r}\left(z_{n}\right)$. Then for all $s \in \mathcal{S}_{r}\left(z_{n}\right)$, we have

$$
s t-\lim _{j} \sum_{n=1}^{\infty} a_{j n}\left\|Y_{j}\left(G_{s} ; .\right)-G_{s}\right\|_{S_{r}\left(z_{n}\right)}=0,
$$

if and only if $s \circ s_{\circ}=1, G_{s}(s \circ \tau)=\tau^{\lambda} s, G_{s}(s \circ v)=s \circ \gamma_{\tau-1}, \ni s_{\circ}, \tau, v \in G_{s i} ; i=0,1,2$; such that $s t-$ $\lim _{j} \sum_{n=1}^{\infty} a_{j n}\left\|Y_{j}\left(G_{s i} ; \cdot\right)-G_{s i}\right\|_{S_{r}\left(z_{n}\right)}=0 ; i=0,1,2$, and $G_{s i}$ is a subgroup from $G_{s} ; i=0,1,2$.

Theorem 1.11 (Al-Muhja, M., 2015) If $\left(z_{n i}\right)_{i=0}^{\infty}$ is defined by (1), then there exists a set $\mathfrak{J}=\left\{z_{n 0}<z_{n 1}<\cdots<z_{n m}<\right.$ $\cdots\} \subseteq \mathbb{N}$, such that $\delta_{\theta}(\mathfrak{J})=1$ and $Y_{j}$ homogeneous group if and only if a sequence $Y=\left(Y_{j}\right)$ is lacunary statistically convergence to $L$.

Now, we present the following result:

Theorem A If $\left(z_{n i}\right)_{i=0}^{\infty}$ is defined by (1), then there exists a set $\mathfrak{J}=\left\{z_{n 0}<z_{n 1}<\cdots<z_{n m}<\cdots\right\} \subseteq \mathbb{N}$, such that $\delta_{\theta}(\mathfrak{S})=1$ and a sequence $Y=\left(Y_{j}\right)$ is bounded statistically lacunary summable to $L$ if and only if it is strongly $\theta_{q}$-convergence to $L$.

\section{Proof of Theorem A and Concluding}

In this section, we want the prove Theorem A, in order to get the installed results in figure 1, it is called $\Omega$ striped.

Firstly (Freedman, A. R. \& Sember, J. J., 1981), we recall the strong convergence fields of various summability methods. In recent years, using statistical A-summability, Riesz's functional supremum formula via statistical limit and Rouche's sequence is B-statistical A-summability (Edely, O. H., \& Mursaleen, M., 2009; Al-Muhja, M., 2014; Al-Muhja, M., Khrajan, M. \& Abdul Hussein, H. J., 2015). 


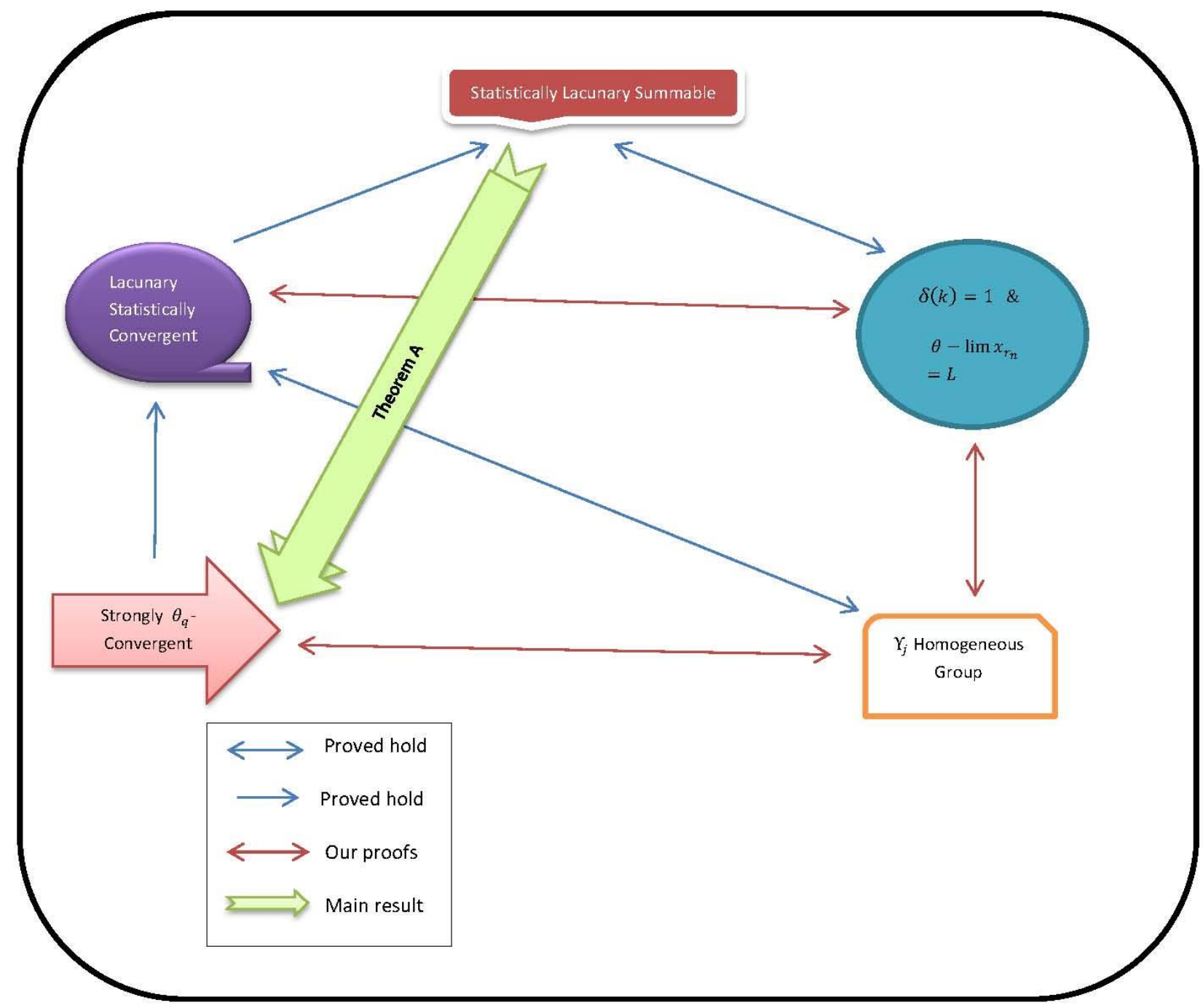

Figure 1. Statistically lacunary summability (SLS) and it is called $\boldsymbol{\Omega}$ striped. There are new results for Korovkin theorem.

Now, we prove our theorem:

Proof of Theorem A. Assume that $Y=\left(\Upsilon_{j}\right)$ is bounded strongly $\theta_{q}$-convergent to limit $L$, since $Y=\left(Y_{j}\right)$ is lacunary statistically convergent to $L$ (Fridy, J. A. \& Orhan, C., 1993), implication $Y=\left(Y_{j}\right)$ is statistically lacunary summable to $L$.

Now, assume that $Y=\left(Y_{j}\right)$ is statistically lacunary summable to $L$, then $\left|t_{r}(x)-L\right| \rightarrow 0$. Hence,

$\frac{1}{h_{r}} \sum_{j \in I_{r}}\left|x_{j}-L\right| \leq \frac{1}{h_{r}} \sum_{j \in I_{r}}\left|x_{j}-L\right|^{q}$, ((Al-Muhja, M. \& Bhaya, E. S., 2010), theorem 2.4), the inquality become:

$$
\leq\left|\frac{1}{h_{r}} \sum_{j \in I_{r}}\left(x_{j}-L\right)\right|^{q}=\left|\frac{1}{h_{r}} \sum_{j \in I_{r}} x_{j}-L\right|^{q}
$$

$$
=\left|t_{r}(x)-L\right|^{q} \rightarrow 0 \text {, as } r \rightarrow \infty \text {, see Definition 1.2, } t_{r}(x) \text { is mean the generalized de la Valle-Poussin. }
$$

Hence $x_{k} \rightarrow L\left[C_{\theta}\right]_{q}$. This is a complete proof.

Corollary B. A sequence $Y=\left(Y_{j}\right)$ is bounded strongly $\theta_{q}$-convergence to $L$ if and only if there exists a set $\mathfrak{J}=$ $\left\{z_{n 0}<z_{n 1}<\cdots<z_{n m}<\cdots\right\} \subseteq \mathbb{N}$, such that $\delta_{\theta}(\mathfrak{S})=1$ and $Y_{j}$ homogeneous group. 
Proof. Suppose $Y=\left(Y_{j}\right)$ is bounded strongly $\theta_{q}$-convergence to $L$. Then from (Mursaleen, M. \& Alotaibi, A., 2011; Theorem 2.2) and theorem 1.11, we have Figure 2. (a). Now, assume that $Y_{j}$ homogeneous group. Then from theorem 1.11, (Mursaleen, M. \& Alotaibi, A., 2011; Theorem 2.1) and Theorem A, we have Figure 2. (b). This is a complete proof.

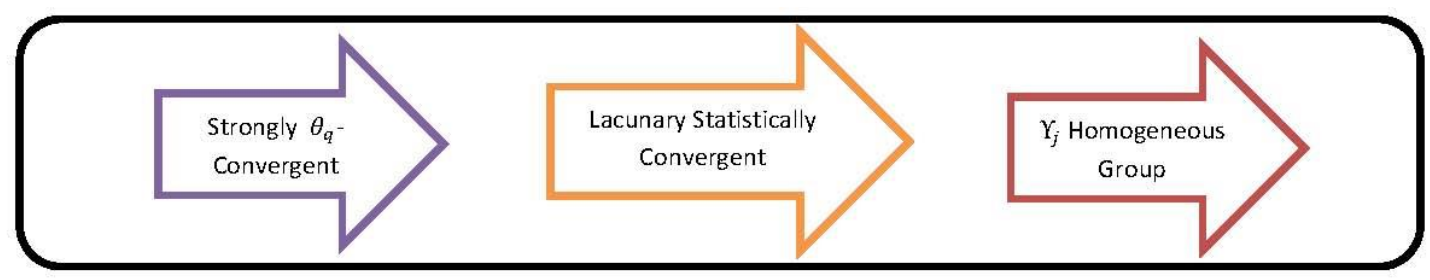

(a)

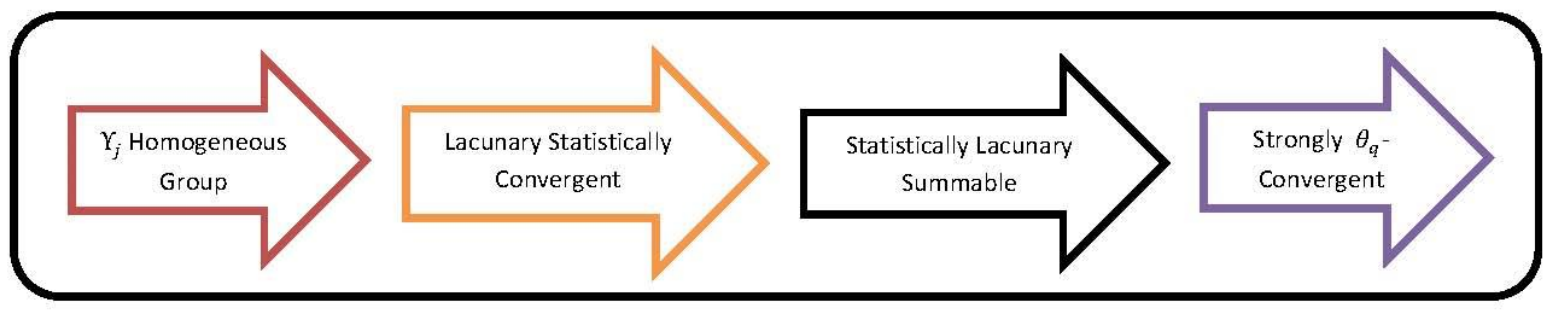

(b)

Figure 2. Proof of Corollary B

Corollary C. A sequence $Y=\left(\Upsilon_{j}\right)$ is statistically lacunary summable to $L$ if and only if $\Upsilon_{j}$ homogeneous group.

Proof. Suppose $Y=\left(Y_{j}\right)$ is statistically lacunary summable to $L$. Then from Theorem A and Corollary B (first condition), we have Figure 3. (a). Now, assume that $Y_{j}$ homogeneous group. Then from theorem 1.11 and (Mursaleen, M. \& Alotaibi, A., 2011; Theorem 2.1), we have Figure 3. (b). This is a complete proof.

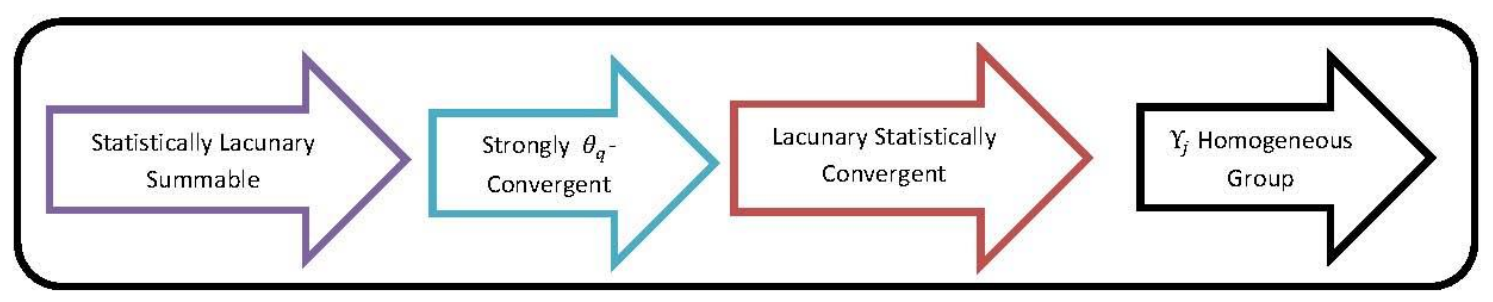

(a)

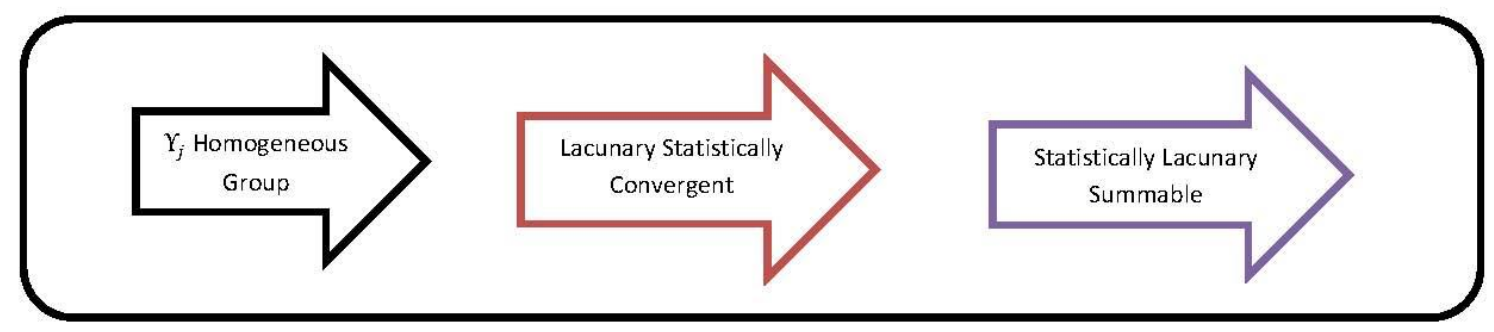

(b)

Figure 3. Proof of Corollary C 
For an $Y=\left(Y_{j}\right)$ sequence is bounded. The following conditions are equivalent:

(SLS 1) Statistically lacunary summable to $L$.

(SLS 2) A set $\mathfrak{J}=\left\{z_{n 0}<z_{n 1}<\cdots<z_{n m}<\cdots\right\} \subseteq \mathbb{N}$, such that $\delta_{\theta}(\mathfrak{J})=1$ and $\theta-\lim x_{r_{n}}=L$.

(SLS 3) $Y_{j}$ homogeneous group.

(SLS 4) Strongly $\theta_{q}$-convergence to $L$.

(SLS 5) Lacunary statistically convergence to $L$.

\section{Acknowledgements}

The authors are grateful for hospitality at the University of Kufa. They thank his fellows for the fruitful discussions while preparing this paper. The authors are thankful to the referees for their careful reading and making some helpful comments which have essentially improved the presentation of this paper.

\section{References}

Al-Muhja, M. (2014). A Korovkin type approximation theorem and Its applications. Abstract and Applied Analysis, vol. 2014, Article ID 859696, 6 pages. https://doi.org/10.1155/2014/859696

Al-Muhja, M. (2015). A spline group - Korovkin approximation theorem. Journal of Mathematics Research, 7(2). https://doi.org/10.5539/jmr.v7n2p110

Al-Muhja, M., \& Bhaya, E. S. (2010). On K-monotone approximation in Lp. Journal of Kufa for Mathematics and computer, 1(1), 98-103.

Al-Muhja, M., Khrajan, M., \& Abdul Hussein, H. J. (2015). An extension of Rouche's theorem via a Korovkin type theorem. European Journal of Scientific Research, 136(1), 107-113.

Edely, O. H., \& Mursaleen, M. (2009). On statistical A-summability. Mathematical and Computer Modeling, 49, 672-680. https://doi.org/10.1016/j.mcm.2008.05.053

Freedman A. R., \& Sember, J. J. (1981). Densities and summability. Pacific Journal of Mathematics, 95(2), 293-305. https://doi.org/10.2140/pjm.1981.95.293

Fridy, J. A., \& Orhan, C. (1993). Lacunary statistical convergence. Pacific Journal of Mathematics, 160(1), 43-51. https://doi.org/10.2140/pjm.1993.160.43

Kopotun, K. A. (2006). On equivalence of moduli of smoothness of splines in Lp, $0<\mathrm{p}<1$. Journal of Approximation Theory, 143, 36-43. https://doi.org/10.1016/j.jat.2006.03.001

Mursaleen, M., \& Alotaibi, A. (2011). Statistical lacunary summability and a Korovkin type approximation theorem. Annali dell' Universita'di Ferrara, 57(2), 373-381. https://doi.org/10.1007/s11565-011-0122-8

Mursaleen, M., \& Alotaibi, A. (2011). Statistical summability and approximation by de la Vallee-Poussin, Applied Mathematics Letter, 24, 320-324. https://doi.org/10.1016/j.aml.2010.10.014

\section{Copyrights}

Copyright for this article is retained by the author(s), with first publication rights granted to the journal.

This is an open-access article distributed under the terms and conditions of the Creative Commons Attribution license (http://creativecommons.org/licenses/by/4.0/). 